\title{
Radioactive boron beams produced by isotope online mass separation at CERN-ISOLDE
}

\author{
J. Ballof ${ }^{1,3, a}$, C. Seiffert ${ }^{1,2}$, B. Crepieux ${ }^{1}$, Ch.E. Düllmann ${ }^{3,7,8}$, M. Delonca ${ }^{1}$, M. Gai ${ }^{6}$, A. Gottberg ${ }^{1, b}$, T. Kröll ${ }^{2}$, \\ R. Lica ${ }^{1,11}$, M. Madurga Flores ${ }^{1}$, Y. Martinez Palenzuela ${ }^{1,9}$, T.M. Mendonca ${ }^{1}$, M. Owen ${ }^{1}$, J.P. Ramos ${ }^{1,4}$, S. Rothe ${ }^{1}$,

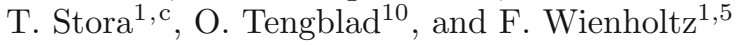 \\ 1 CERN, 1211 Geneva 23, Switzerland \\ 2 Technische Universität Darmstadt, Schlossgartenstr. 9, 64289 Darmstadt, Germany \\ 3 Johannes Gutenberg - Universität Mainz, Institut für Kernchemie, Fritz-Strassmann-Weg 2, 55128 Mainz, Germany \\ 4 Laboratory of Powder Technology, École Polytechnique Fédérale de Lausanne (EPFL), Lausanne, CH-1015, Switzerland \\ ${ }^{5}$ Universität Greifswald, Domstraße 11, 17489 Greifswald, Germany \\ 6 LNS at Avery Point, University of Connecticut, Groton, CT 06340, USA \\ 7 GSI Helmholtzzentrum für Schwerionenforschung, 64291, Darmstadt, Germany \\ 8 Helmholtz-Institut Mainz, 55099 Mainz, Germany \\ 9 KU Leuven, Instituut voor Kern- en Stralingsfysica, B-3001 Leuven, Belgium \\ 10 Instituto de Estructura de la Materia, CSIC, Serrano 113 bis, ES-28006 Madrid, Spain \\ 11 "Horia Hulubei" National Institute of Physics and Nuclear Engineering, RO-077125, Bucharest, Romania
}

Received: 26 August 2018 / Revised: 31 January 2019

Published online: 13 May 2019

(C) The Author(s) 2019. This article is published with open access at Springerlink.com Communicated by K. Blaum

\begin{abstract}
We report on the development and characterization of the first radioactive boron beams produced by the isotope mass separation online (ISOL) technique at CERN-ISOLDE. Despite the long history of the ISOL technique which exploits thick targets, boron beams have up to now not been available. This is due to the low volatility of elemental boron and its high chemical reactivity which make the definition of an appropriate production target unit difficult. In addition, the short half-lives of all boron radioisotopes complicate tracer release studies. We report here on dedicated offline release studies by neutron capture and alpha detection done with implanted ${ }^{10} \mathrm{~B}$ in prospective target materials, as well as molecule formation and ionization tests, which suggested the use of multiwalled carbon nanotubes (CNT) as target material and injection of sulfur hexafluoride $\mathrm{SF}_{6}$ to promote volatile boron fluoride formation. Two target units equipped with an arc discharge electron impact ion source VADIS coupled to a water cooled transfer line to retain non-volatile elements and molecules were subsequently tested online. The measured yield of these first ${ }^{8} \mathrm{~B}$ ISOL beams increases in the series ${ }^{8} \mathrm{BF}_{3}<{ }^{8} \mathrm{BF}<{ }^{8} \mathrm{~B}<{ }^{8} \mathrm{BF}_{2}$, reaching a maximum yield of $6.4 \times 10^{4}{ }^{8} \mathrm{BF}_{2}{ }^{+}$ions per $\mu \mathrm{C}$ of protons.
\end{abstract}

\section{Introduction}

At CERN-ISOLDE [1] more than 1000 radioisotopes of 74 different chemical elements can be delivered to a large spectrum of experimental setups for investigations in nuclear structure, atomic physics and applications. These isotopes are produced by the ISOL (Isotope mass Separation OnLine) method inside a combined target and ion source unit upon impact of the $1.4 \mathrm{GeV}$ proton driver

\footnotetext{
a e-mail: jochen.ballof@cern.ch

b Present address: TRIUMF, 4004 Wesbrook Mall, Vancouver, BC V6T 2A3, Canada.

c e-mail: thierry.stora@cern.ch (corresponding author)
}

beam from the PS-Booster. The yield of radioactive isotopes which can be extracted from ISOL-like facilities is strongly dependent on the half-life and the chemical properties of the desired element. For some elements (e.g., Na, $\mathrm{K}$ ) extraction efficiencies from ISOL targets can be close to $100 \%$, while for other elements their extraction has proven difficult or even impossible up to now, despite decades of research in this field. Examples for isotopes with low extractable yields are refractory or chemically very reactive elements such as boron, tungsten or rhenium. The reasons why yields can be very low is apparent from the extraction process itself [2]. After isotope production typically through fission, fragmentation or spallation nuclear reactions, the neutral isotopes have to diffuse to the surface of 
the target material grain where they can evaporate or react to form more volatile molecules, in the case of a molecular extraction. During the subsequent migration through the target setup towards the ion source, the isotope experiences numerous interactions with the surrounding target and structural material. At each interaction losses due to chemical reactions or irreversible sticking on the surfaces can occur. In the case of boron these losses are so large, that no Radioactive Ion Beam (RIB) was produced by the ISOL method until now, although many possibilities are offered by different combinations of targets and ion sources operated in facilities such as ISOLDE at CERN. In the present article, we introduce the different developments made, and the description of the target unit obtained for the delivery of ${ }^{8} \mathrm{~B}$ as molecular ion beam [3]. Finally we report on the online results obtained at ISOLDE with the prepared target unit.

\section{ISOL beam extraction processes}

The extractable yield $Y$ of isotopes produced in an ISOLDE target unit can be estimated by folding the extraction probability of a single isotope weighted by the number of initially produced isotopes $N_{0}$ per $\mu \mathrm{C}$ of primary beam with the distribution function $p(t)$ of extraction times in the investigated setup:

$$
\begin{aligned}
Y & =\int_{0}^{\infty} N(t) p(t) \mathrm{d} t, \quad \text { where } \\
N(t) & =N_{0} e^{-\lambda t} \epsilon_{\text {irrev }} \epsilon_{\text {form }} \epsilon_{\text {ion }}, \quad \text { and } \\
t & =t_{\text {diff }}+t_{\text {ads }}+t_{\text {eff }} .
\end{aligned}
$$

Here, $\lambda$ is the decay constant of the isotope, $t_{\text {diff }}$ is the diffusion time out of the material, $t_{\text {ads }}$ the delay due to sticking on surfaces, $t_{\text {eff }}$ the time of flight through the target container and into the ion source and $\epsilon_{\text {irrev }}$ is the transport efficiency considering chemical losses and irreversible adsorption on surfaces. $N(t)$ is the yield of extracted isotopes, which need time $t$ to propagate from the target material to the ion source. The expression holds for pulsed and continuous primary beam. However, modified expressions for facilities using a continuous driver beam are typically used, which give the radioactive beam intensity as number of radioactive ions per second. If the vapor pressure of a desired element is too small at achievable temperatures, extraction as a more volatile molecule is necessary. The formation efficiency for a particular molecular sideband is given by $\epsilon_{\text {form }}$. Finally, $\epsilon_{\text {ion }}$ gives the ionization efficiency.

Diffusion in solid grains of the target material as well as effusion processes through open space are responsible for the shape of the distribution function $p(t)$, which holds for a stable, non-decaying isotope [4].

The initial number of produced isotopes $N_{0}$ is determined by the production cross section, proton beam intensity and number of target atoms. Since in most cases, the geometry of the target is predefined, a high target material density is favorable to increase $N_{0}$. However, a high density might be adverse for the diffusion of the isotope out of the material. A high porosity and small grain size is desired to allow fast and efficient diffusion and migration out of the target material [5]. If isotopes need to be extracted as molecules, the target setup can be equipped with additional gas injection lines, which allows to provide reaction partners for the formation of volatile carrier molecules. To minimize the losses due to sticking and chemical reaction of the reaction products, each of the materials used in the entire target and ion source assembly has to be considered separately.

Although the variety of available setups and advances in target materials production allows the extraction of isotopes of a large range of elements, no beams of radioactive boron were extracted at any thick-target ISOL facility prior to the development reported here. The experimental interest in beams of ${ }^{8} \mathrm{~B}$ is manifold: besides interest in studying the proton halo structure $[6]$ or high lying resonances of ${ }^{9} \mathrm{C}$, which become accessible by elastic scattering of ${ }^{8} \mathrm{~B}$ on a proton target [7], beams of boron would be ideal to investigate diffusion properties in semi conductors where boron is commonly used as a dopant [8]. Recently beams of ${ }^{8} \mathrm{~B}$ were extracted at KVI at a rate of 7 ions per second [9] and at the thin-target facility IGISOL at a rate of 200 ions per second [10]. However, the yields available in these facilities are not sufficient for the above mentioned experiments, which require rates in the order of $5 \times 10^{3}$ ions per second or above, and in some cases post acceleration of the beam. The relatively high production cross sections combined with the thick-target technologies available at ISOL facilities, like ISOLDE, open up new perspectives for physics experiments, if control over the boron release out of the target material and ionization can be gained.

In this work the factors important for the extraction (cf. eq. (1)) of radioactive boron $\left({ }^{8} \mathrm{~B}, T_{1 / 2}=770 \mathrm{~ms}\right.$ ) are investigated. This includes the choice of target material based on their production cross sections and the experimental determination of boron mobility in candidate targets, taking into account overall diffusion and effusion release processes. A dedicated offline release experiment was conducted exploiting the high neutron capture cross section of ${ }^{10} \mathrm{~B}$ to determine which material allows a fast extraction and therefore high mobility. Since boron exhibits not only a high chemical reactivity but also a high boiling point, it needs to be extracted as molecular sideband. Upon reaction with a fluorinating agent, boron readily forms volatile and relatively inert fluorides. In comparison to other halogens, fluorine possesses only one stable isotope which allows to concentrate the molecular formation on a single isobar and therefore to achieve a higher mass separation efficiency. In a second step, the release of boron as boron fluorides $\left(\mathrm{BF}_{\mathrm{x}}, 0 \leqslant \mathrm{x} \leqslant 3\right)$ was studied using an ISOLDE target setup and the offline isotope mass separator. This allowed to compare the measured beam composition with chemical equilibrium calculations performed under different conditions of $\mathrm{SF}_{6}$ injection as well as the investigation of ionization behavior and influence of target parameters on the beam formation. Following up on the positive outcome of the preparatory experiments, a prototype target could be built, and beams of ${ }^{8} \mathrm{~B}$ were produced online and characterized. 
Table 1. Nominal densities [11] and typical material densities of prospective target materials for the production of boron radioisotopes at ISOLDE.

\begin{tabular}{c|c|c} 
Material & $\begin{array}{c}\text { Nominal density } \\
{\left[\mathrm{g} \mathrm{cm}^{-3}\right]}\end{array}$ & $\begin{array}{c}\text { ISOLDE density } \\
{\left[\mathrm{g} \mathrm{cm}^{-3}\right]}\end{array}$ \\
\hline $\mathrm{CaO}$ & 3.34 & $0.38^{\mathrm{a}}$ \\
$\mathrm{CNT}$ & $2.2^{\mathrm{c}}$ & $0.4^{\mathrm{b}}$ \\
$\mathrm{HfO}_{2}$ & 9.68 & - \\
$\mathrm{CaF}_{2}$ & 3.18 & - \\
$\mathrm{Y}_{2} \mathrm{O}_{3}$ & 5.03 & $3.16^{\mathrm{b}}$ \\
$\mathrm{Al}_{2} \mathrm{O}_{3}$ & 3.99 & -
\end{tabular}

${ }^{\mathrm{a}}$ Value taken from ref. [5].

b Density of available materials, which were also used in the offline mobility studies (sect. 4).

c The theoretical density of graphite is given in the case of multiwalled carbon nanotubes (CNT).

\section{In-target isotope production}

The in-target production of the desired isotope is the first parameter that needs to be considered for the development of new ISOL beams. The factors important for a high production rate are the production cross section $\sigma$ for the particular isotope, the number of target nuclei and the number of protons impinging on the target. In this work, the production cross section of boron isotopes by bombarding a selection of target materials with $1.4 \mathrm{GeV}$ protons was calculated using the ABRABLA code [12]. The selection of target materials is based on characteristics like melting point and chemical compatibility. Here the production cross sections were calculated for graphite, aluminum oxide, calcium oxide, calcium fluoride, hafnium oxide and yttrium oxide.

From the production cross section $\sigma$ the number of produced isotopes $N_{0}$ which is used in eq. (1) can be calculated via the equation

$$
N_{0}=\sigma N_{p} N_{T}
$$

where $N_{p}$ is the number of protons impinging on the target and $N_{T}$ the areal density of target atoms, given by the dimensions of the target container and the density $\rho$ of the target material. For the sake of comparison the nominal densities of the bulk materials were used for the calculation of the in-target production. As discussed in sect. 2 the practical densities of materials suitable for ISOL targets are lower to achieve a desired open porosity. Both densities are presented in table 1 and the in-target yields in isotopes per $\mu \mathrm{C}$ of proton beam according to eq. (2) are displayed in fig. 1 , which take into account the dimensions of the cylindrical ISOLDE target container, having length and diameter of $20 \mathrm{~cm}$ and $2 \mathrm{~cm}$, respectively [13].

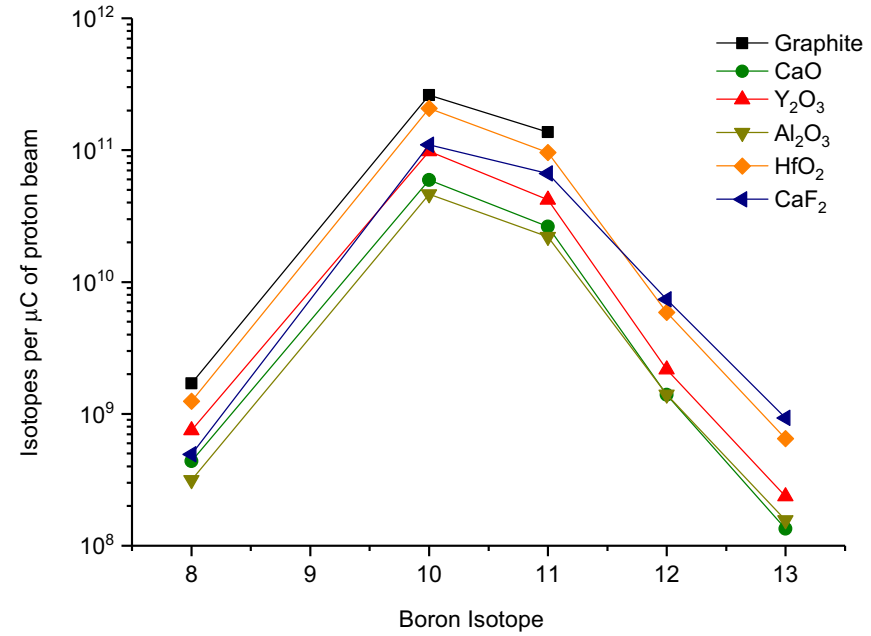

Fig. 1. In-target production yields of boron isotopes in potential target materials for the extraction of radioactive boron beams, obtained with the ABRABLA code. At ISOLDE, an average proton intensity of up to $2 \mu \mathrm{A}$ is available. Nominal densities, as given in table 1 are assumed.

\section{Mobility of boron in potential target materials}

The diffusion process is specific for every combination of target material and diffusing element. The diffusion coefficient $D$ is a measure for the mean displacement of a particle per time unit and exponentially increases with the temperature as given by the Arrhenius equation [14]

$$
D=D_{0} \mathrm{e}^{-Q /(\mathrm{R} T)},
$$

where $D_{0}$ is a temperature independent pre-exponential factor, $Q$ the activation energy, R the universal gas constant and $T$ the absolute temperature. The characteristic diffusion time is given by $\tau_{D}=a^{2} /\left(\pi^{2} D\right)[15]$.

ISOLDE targets can be operated at temperatures up to about $T_{\max } \approx 2500 \mathrm{~K}$. However, in many cases target materials start to degrade due to sintering effects already at lower temperatures which can result in an increase of the characteristic diffusion time and thereby reducing the yield of the extracted radioactive ions [5]. As diffusion is a slow process in comparison to migration through open space, a high open porosity and a small grain size are the desired characteristics of target materials. Determining diffusion coefficients is often difficult and requires sophisticated setups. For the production of radioactive ion beams the combination of both diffusion and migration through open space is important and can be summarized as the mobility of an element in the target.

In a first approximation, diffusion can be described by Fick's second law, and solutions for certain geometries and boundary conditions are provided in ref. [15]. Effusion effects are negligible for long heat treatment times (see sect. 7.1), and the fraction of isotopes remaining in the 
solid after a heat treatment time $t$ computes to

$$
f(t)=\frac{2 n}{\pi^{2}} \sum_{m=1}^{\infty} c_{m}^{-1} e^{-c_{m} t / \tau_{D}},
$$

with $c_{m}=\left(j_{0, m} / \pi\right)^{2}$ for fibers $(n=2)$, and $c_{m}=m^{2}$ for spheres $(n=3) \cdot j_{0, m}$ stands for the $m$-th positive root of the Bessel function of order zero and $\tau_{D}$ is the characteristic diffusion time. The latter can be deduced, if $f(t)$ was determined in an experiment. The solution of the diffusion differential equation given in eq. (4) was derived in ref. [15] under the boundary condition, that the diffusing species is uniformly distributed in the sample. However, after a heat treatment, the originally uniform distribution profile is disturbed due to depletion of the particle density near the edges of each grain. For subsequent heat treatments, the mean path a particle needs to travel to reach the surface of the grain, is longer compared to the original distribution. Within the mathematical procedure to correct for the disturbance of the profile, the two conducted heat treatments at different temperatures are replaced by one heat treatment at the higher temperature. The replacement procedure to derive the characteristic diffusion time for the second treatment, is adjusted such that the release fraction at the beginning and the end match the experimental treatment. For a discussion see ref. [16].

In earlier studies of boron diffusion in different materials by neutron depth profiling, more complex phenomena have been found. In certain matrices, accumulation of boron on the surface of the sample and propagation of the apparent inventory towards the surface of the sample were observed. The latter was attributed to the presence of radiation induced damage, introduced during the implantation process [17].

For this work the mobility of boron in different potential target materials was studied. The high cross section of $3.84 \mathrm{~kb}$ for the reaction ${ }^{10} \mathrm{~B}(n, \alpha){ }^{7} \mathrm{Li}$ at thermal neutron energies [18] offers an elegant way to determine the amount of boron tracers and therefore to study the mobility of boron in materials. The branching ratio and expected particle energies for this reaction are as follows $[19,20]$ :

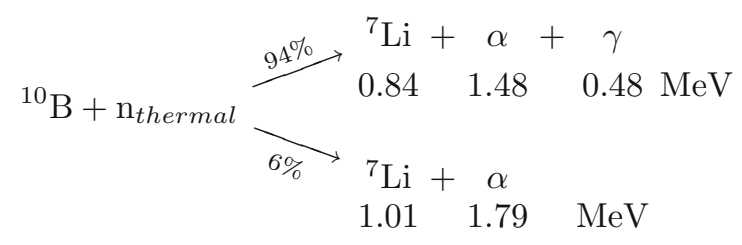

The mobility of boron in three out of the six materials considered in the previous chapter was studied. We included yttrium oxide $\left(\mathrm{Y}_{2} \mathrm{O}_{3}\right)$ as a representative class for the oxide materials as it had appropriate nanometric grain size and porosity, and two carbon allotropes namely graphite and multiwalled carbon nanotubes (CNT). To obtain reproducible and representative results, calcium oxide, for instance in the form of a nanoporous material, needs to be handled under inert gas atmosphere [5], and was therefore not considered for practical purposes. $\mathrm{CaF}_{2}$ was neither considered following the outcome of chemical equilibrium calculations as discussed in sect. 5. Moreover, the mobility of boron in $\mathrm{MgF}_{2}$ has already been studied, which might serve as surrogate for $\mathrm{CaF}_{2}$ [21].

Samples for the three materials were prepared in pressed powder form (pellets) and $4 \times 10^{16}$ atoms of ${ }^{10} \mathrm{~B}$, extracted as $\mathrm{BF}_{2}+$ from an ISOLDE target unit (cf. sect. 6) were implanted in each pellet at the ISOLDE offline mass separator at $50 \mathrm{kV}$ extraction voltage. In the case of CNT and Yttria, the sample was biased additionally $(-12 \mathrm{kV})$ to increase the implantation depth. Each sample was subjected to neutron irradiation with an intense ${ }^{238} \mathrm{Pu}$-Be source of $1 \mathrm{Ci}$ available from the CERN dosimetry service, and simultaneously the resulting $\alpha$-particles $(1.48 \mathrm{MeV})$ were detected using silicon detectors. A determination of the initial boron inventory with this method was done after preparation of each sample. Subsequently, the samples were heat treated to promote the diffusion process and the remaining fraction of boron atoms in the sample was determined. The heat treatment and measurement were repeated to a maximum temperature of $2000^{\circ} \mathrm{C}$, or until no boron could be detected anymore.

\subsection{Materials}

Multiwalled carbon nanotubes were supplied by Nanocyl, ref. $\mathrm{NC} 3100,>95 \%$ purity, $10 \mathrm{~nm}$ diameter, $1.5 \mu \mathrm{m}$ length. Commercially available Yttria (Alfa Aesar, yttrium (III) oxide, $99.995 \%$ (REO) 25-50 nm APS Powder) and graphite (Alfa Aesar, ref. 40798, 325 mesh, $<44 \mu \mathrm{m}$ ) were used. The carbon materials have been characterized and results are provided in ref. [22].

\subsection{Experimental setup and measurement}

The detection of $\alpha$-particles was achieved using an Ortec Alpha Aria spectrometer equipped with an Ortec silicon detector $\left(450 \mathrm{~mm}^{2}, 100 \mu \mathrm{m}\right.$ depletion layer $)$. The sample was placed at a distance of $5 \mathrm{~mm}$ to the detector and exposed to thermalized neutrons from the ${ }^{238} \mathrm{Pu}-\mathrm{Be}$ source. The emerging fast neutrons were moderated by a $7 \mathrm{~cm}$ polyethylene (PE) block. In addition $15 \mathrm{~cm}$ of lead served as shielding to suppress background due to gamma radiation. After measurement of the $\alpha$-particle rate, and subsequent deduction of the apparent boron inventory, the samples were heated in a tantalum furnace, similar to a standard target container [13].

To investigate the temperature dependence of the diffusion, samples were heated for 30 minutes in 5 (4 for CNT) steps, from room temperature to approximately $2000^{\circ} \mathrm{C}$. After each step, samples were exposed to the neutron source for approximately 50 hours and the emitted $\alpha$-particles were recorded.

\subsection{Results and discussion}

A typical spectrum obtained from the silicon detector within a measurement time of 27 hours is shown in fig. 2 . 


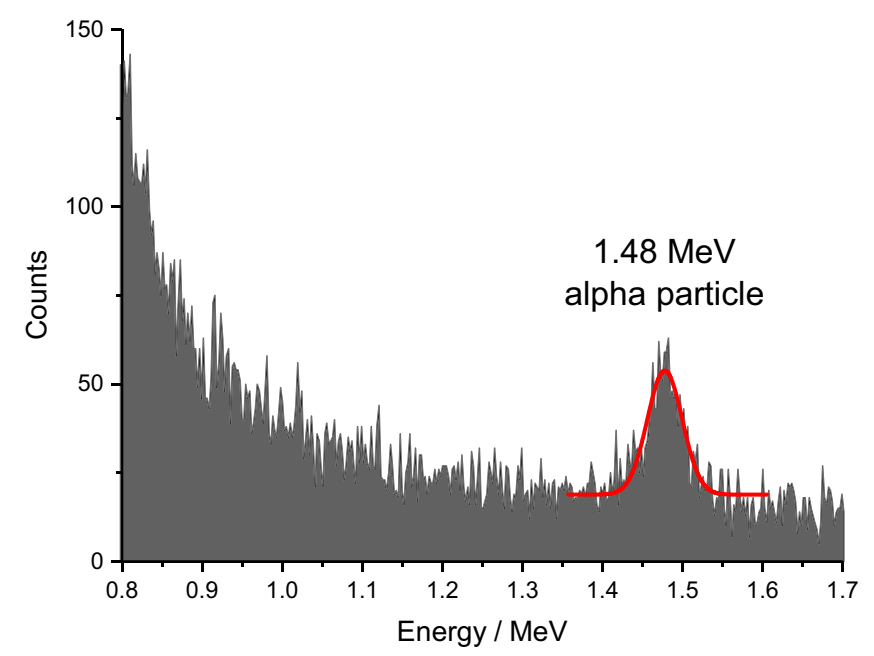

Fig. 2. Typical silicon detector spectrum obtained within a measurement time of 27 hours for the offline mobility studies. The peak arising from the $1.48 \mathrm{MeV}$ alpha particle was fitted with a Gaussian function.

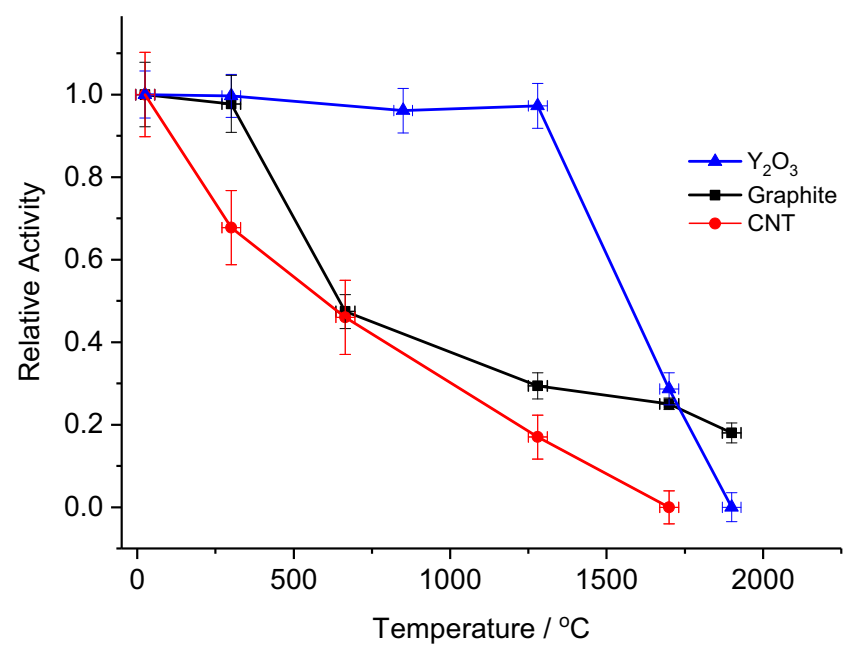

Fig. 3. Relative apparent boron inventory of the samples measured in the ${ }^{10} \mathrm{~B}(n, \alpha)^{7} \mathrm{Li}$ reaction, after successive offline mobility studies (see text).

Due to limited statistics, only the peak arising from the $1.48 \mathrm{MeV}$ alpha particle is detectable. Peak center and area were obtained by fitting with a Gaussian function. Within the errors of the fits, which were less than $\pm 0.05 \mathrm{MeV}$ for the peak center energy, we did not observe a reduction or increase of the alpha energy after subjecting the respective samples to the heat treatments. Hence we assume, that in-diffusion into deeper layers is not the primary reason for the depletion of the apparent boron inventory. Figure 3 shows the apparent boron inventory evolution of the samples obtained from the peak areas. To ease comparison, measurements are normalized to the initial implantation of each sample. The boron depletion is believed to be caused by its migration to the surface of the material, where it can desorb and is expected to be caught on parts of the tantalum oven or extracted as volatile species. Further studies would be necessary to fully explain the desorption process. We believe, that the desorption process is promoted by reactions with residual gases. Candidate compounds are oxides, oxohalides or halides. Oxygen is available as residual gas $\left(5 \times 10^{-5} \mathrm{mbar}\right)$, and might react with boron to form boron monoxide or dioxide as intermediate carriers, which have been identified in other processes [23]. Halogens might be present as impurities in the oven or host material. A certain amount of fluorine was introduced during the sample preparation by implantation of $\mathrm{BF}_{2}{ }^{+}$.

It is obvious, that the diffusion behavior of boron significantly varies within the set of investigated host materials. Diffusion of boron in single crystals of graphite has been studied by Henning [24], who found almost 1000 times higher diffusion constants for diffusion parallel to the carbon layers compared to the diffusion perpendicular to the layers. At a temperature of $2291^{\circ} \mathrm{C}$, the diffusion constant was determined to be $3.9 \times 10^{-10} \mathrm{~cm}^{2} / \mathrm{s}$, if diffusion occurs parallel to the carbon layers. Novak et al. investigated the diffusion in polycrystalline graphite and obtained diffusion coefficients as high as $3.1 \times 10^{-6} \mathrm{~cm}^{2} / \mathrm{s}$ at $2200{ }^{\circ} \mathrm{C}$ [25]. Despite our setup not allowing to derive diffusion constants, the cited values indicate fast diffusion and a strong dependence on the allotrope, which is reflected in the results shown in fig. 3. After the heat treatment at $700{ }^{\circ} \mathrm{C}$ the amount of boron in the graphite sample drops from almost $100 \%$ to about $50 \%$ of the initially implanted amount. Heating the graphite sample to $1300^{\circ} \mathrm{C}$ causes an additional drop of remaining boron to $30 \%$. Increasing the temperature further does not change the remaining amount significantly which suggests that the remaining boron is physically or chemically confined in the graphite matrix. The situation for multiwalled carbon nanotubes is slightly different. Similar to the graphite sample, significant fractions are released at relatively low temperatures with a decrease to approximately $65 \%$ after heating to a temperature of $300^{\circ} \mathrm{C}$. Further heating shows a faster decrease of boron in comparison to graphite. After heating to $1700^{\circ} \mathrm{C}$ no remaining boron could be detected during measurements. Applying eq. (4) and a disturbed profile correction yields at the highest respective temperatures, a characteristic diffusion time of $\tau_{D}^{\mathrm{CNT}}=8.6_{-1.5}^{+3.5} \times 10^{2} \mathrm{~s}$ for carbon nanotube fibers at $1700^{\circ} \mathrm{C}$, while a lower limit of 5 times this value was determined for the micron-sized graphite material at $1900^{\circ} \mathrm{C}$, which was treated as agglomeration of particles. The given errors are computed from the uncertainty in the release fraction. The application of eq. (4) requires, that boron is implanted uniformly in the material grain. For carbon nanotubes, an implantation depth of $177 \mathrm{~nm}$ was estimated by SRIM 2013 [26,27]. Considering the nanometric structure, the condition of a uniform profile is assumed to be met (cf. table 2). However, in the case of the more dense and micron-sized graphite, boron is only implanted close to the surface. It is therefore assumed to be released faster in comparison to a uniform distribution, only allowing to give a lower limit of the characteristic diffusion time within the used model. 
Table 2. Raw material and implantation characteristics, expected implantation depth and range of $1.48 \mathrm{MeV}$ alpha particles used in the mobility study. The expected implantation depth and alpha range were obtained by SRIM [26,27].

\begin{tabular}{|c|c|c|c|c|}
\hline \multirow{2}{*}{ Material } & \multirow{2}{*}{ Particle size } & \multicolumn{2}{|c|}{ Implantation } & Alpha \\
\hline & & energy & depth & range \\
\hline $\mathrm{CNT}$ & $9.5 \mathrm{~nm} \times 1.5 \mu \mathrm{m}^{\mathrm{a}}$ & $62 \mathrm{keV}$ & $177 \mathrm{~nm}$ & $22 \mu \mathrm{m}$ \\
\hline Graphite & $5.3 \mu \mathrm{m}^{\mathrm{b}}[22]$ & $50 \mathrm{keV}$ & $35 \mathrm{~nm}$ & $4.0 \mu \mathrm{m}$ \\
\hline $\mathrm{Y}_{2} \mathrm{O}_{3}$ & $67 \mathrm{~nm}^{\mathrm{b}, \mathrm{c}}[28]$ & $62 \mathrm{keV}$ & $51 \mathrm{~nm}$ & $5.4 \mu \mathrm{m}$ \\
\hline
\end{tabular}

a As given by the supplier.

b Particle size parameter obtained by specific surface area.

c Sintering is expected at elevated temperatures [28].

While diffusion in-between and through the walls of the nanotubes should be the same as for graphite, additional diffusion along and across the surface and through the capillaries of the tubes can be expected. This is a probable reason for the faster decrease of boron in the material [25, 29-31].

In the case of Yttria almost no change can be observed up to $1300{ }^{\circ} \mathrm{C}$. The remaining amount of boron at this temperature is close to $100 \%$ of that initially implanted. However, heating the sample to $1700^{\circ} \mathrm{C}$ shows a drop of $50 \%$. After subsequent heating to $1900^{\circ} \mathrm{C}$ no remaining boron was detectable during irradiation with neutrons. However, preliminary investigations show sintering at these temperatures [28], and simulations indicate possible decomposition in vacuum above $1900^{\circ} \mathrm{C}$ [32]. Also taking into account the diffusion properties of boron in $\mathrm{MgF}_{2}$ as given in ref. [21], multiwalled carbon nanotubes offer favorable diffusion characteristics.

\section{Chemical equilibrium considerations}

Besides production cross sections and diffusion inside the target material, chemical processes play an important role in the ISOL thick-target release processes. Boron does not only have a low volatility, but also exhibits a high reactivity towards many materials present in the target assembly. Hence, boron has to be extracted as part of a volatile molecule. The demands on the volatile compound are multi-fold. The formation should be fast, it needs to be stable at operation temperature, inert towards reaction with target materials and ionizable with sufficiently large cross sections.

After an evaluation of the known compounds we have chosen boron trifluoride as volatile carrier and sulfur hexafluoride as fluorinating agent. Both are gases at room temperature. Chemical equilibrium calculations with a set of target materials such as alumina, yttria, magnesium fluoride, calcium fluoride and carbon, have been conducted using the software package HSC 7 [32], based on the included thermodynamic data. The interactions with other materials present in the target assembly, like tantalum and molybdenum were also considered.

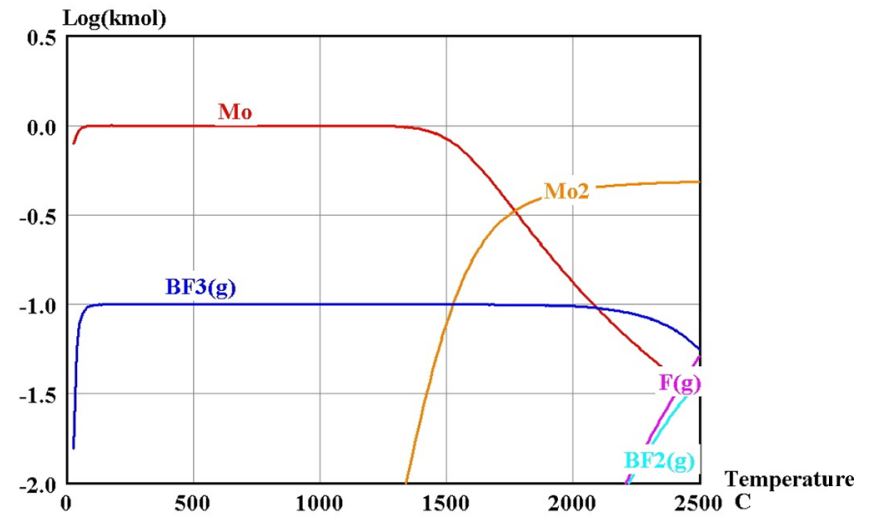

Fig. 4. Chemical equilibrium between molybdenum and $\mathrm{BF}_{3}$.

The results of the calculation performed for molybdenum and $\mathrm{BF}_{3}$ are shown as an example in fig. 4. It can be seen that within the calculated system gaseous boron trifluoride is stable even at high temperatures and does not form thermodynamically favoured compounds with molybdenum. Only at temperatures above $2200^{\circ} \mathrm{C}$ the difluoride starts to build up and eventually becomes the dominating species well above $2500^{\circ} \mathrm{C}$. Similar results were obtained for the equilibrium between other construction materials and $\mathrm{BF}_{3}$.

The common result for all calculations aiming at the formation of $\mathrm{BF}_{3}$ in different target materials is that fluorine has to be available in excess. If this is not the case tantalum borides are expected to be formed. In the case of alumina, volatile molecules of $\mathrm{BF}_{3}$ and $\mathrm{BOF}$ are formed in relatively narrow temperature ranges. Here, $\mathrm{BF}_{3}$ can be expected from $800^{\circ} \mathrm{C}$ to $1400^{\circ} \mathrm{C}$ and $\mathrm{BOF}$ from $1400^{\circ} \mathrm{C}$ to $2100^{\circ} \mathrm{C}$. Furthermore, calculations predict the formation of aluminum borides. The equilibrium with yttria indicates a dominant formation of BOF starting from $1500{ }^{\circ} \mathrm{C}$. Below this temperature no volatile boron species is formed. For calcium fluoride as well as magnesium fluoride formation of boron fluoride is thermodynamically favoured. Unfortunately the temperature at which formation of $\mathrm{BF}_{3}$ starts is, in both cases, higher than the boiling point of the material. For $\mathrm{MgF}_{2}$ gaseous $\mathrm{BF}_{3}$ appears above approximately $1400^{\circ} \mathrm{C}$, for the case of $\mathrm{CaF}_{2}$ above $1600^{\circ} \mathrm{C}$.

The calculations indicate that $\mathrm{BF}_{3}$ is not chemically retained in a carbon-based target material. This, along with the availability of carbon nanomaterials which allow fast diffusion made this selection our preferred choice. Also the very high specific surface favors volatilization by molecule formation with an injected gas.

\section{Ionization behavior and extraction efficiency}

The formation and ionization of boron fluoride ions, socalled sidebands, was tested at the ISOLDE offline separator by placing elemental boron powder in the target container of a typical ISOLDE target. The tantalum container was connected to a VADIS ion source [33] via a water 


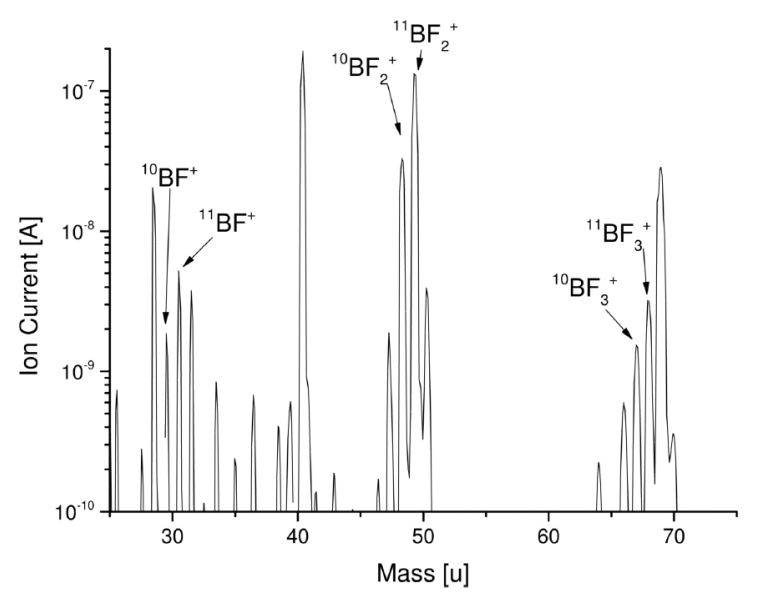

Fig. 5. Mass distribution of boron fluorides ions extracted from a solid boron sample which was placed in heated tantalum container. $\mathrm{SF}_{6}$ was injected through a calibrated leak of $5 \times$ $10^{-5} \mathrm{mbar} \mathrm{L} / \mathrm{s}$ (air).

cooled copper transfer line and equipped with a calibrated leak of $5 \times 10^{-5} \mathrm{mbar} \mathrm{L} / \mathrm{s}$ (air) allowing the injection of $\mathrm{SF}_{6}$. A typical mass spectrum of the extracted beam is shown in fig. 5 . The predominant species is $\mathrm{BF}_{2}{ }^{+}$, which is expected to arise from dissociative ionization of $\mathrm{BF}_{3}$. The container was gradually heated from about $100{ }^{\circ} \mathrm{C}$ to $1600^{\circ} \mathrm{C}$. The ion yield rises from lower temperatures to a maximum at about $1500^{\circ} \mathrm{C}$ and drops again towards higher temperatures. In sect. 4 it was shown that fast diffusion of boron in graphite and CNT is expected in this temperature range, thus the extraction of boron fluoride from a CNT target is also expected.

Besides the dependency of the extracted boron fluorides current on temperature, the dependency on the amount of injected $\mathrm{SF}_{6}$ was studied in the range from $1 \times 10^{-5}$ to $5 \times 10^{-5} \mathrm{mbar} \mathrm{L} / \mathrm{s}$ (air). From equilibrium calculations a linear dependency of the $\mathrm{BF}_{3}$ formation on available fluorine is expected. This correlation was confirmed in the measurements. The extracted ion current of $\mathrm{BF}_{2}{ }^{+}$increases linearly with an increase of injected fluorine. Consequently, a high flow rate of injected sulfur fluoride leads to the most efficient extraction. However, the flow rate is limited by the target station gas pumping capabilities, which have to maintain a pressure below $5 \times 10^{-5}$ mbar on the extraction side to ensure proper operation of the ion source and beam transport.

The calibrated leak allows to determine the combined efficiency for ionization, formation of the compound and transport from target container to ion source, which are $\epsilon_{\text {ion }}, \epsilon_{\text {form }}$ and $\epsilon_{\text {irrev }}$ in eq. (1), respectively. The combined efficiency was evaluated to be $1.5 \%$. While the release studies (sect. 4) were done on a time-scale much longer than the half-life of ${ }^{8} \mathrm{~B}$, making the assessment of the release efficiency difficult, these results lead us to expect that some fraction of the in-target yield would be produced as $\mathrm{BF}_{2}{ }^{+}$molecular ions.

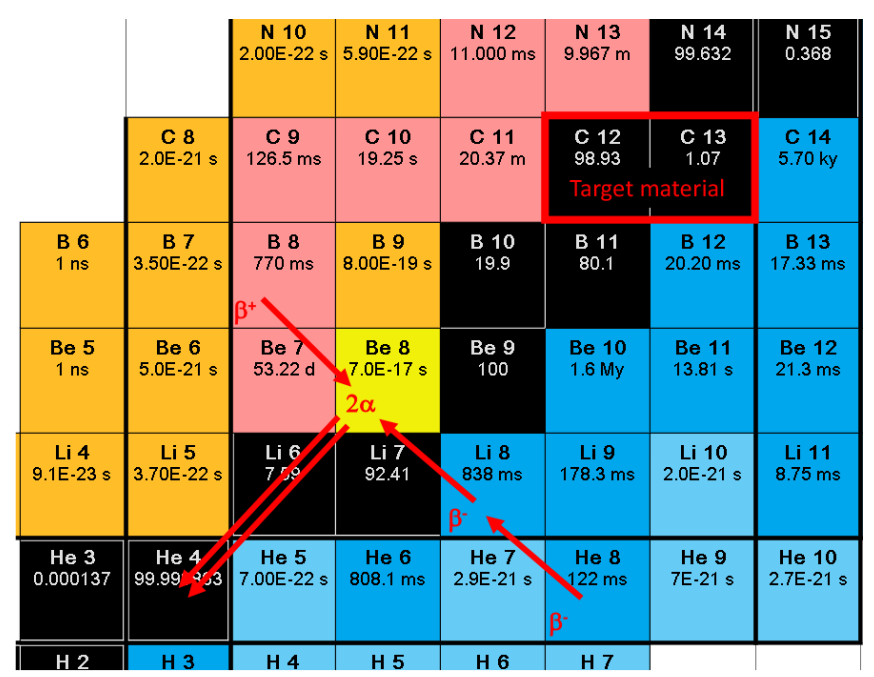

Fig. 6. Cutout of the nuclide chart showing the target material and selected nuclei produced in fragmentation reactions by impact of the $1.4 \mathrm{GeV}$ proton beam and their decay modes. (Adapted from ref. [34]).

\section{Online measurements}

Two target units have been tested in three online runs at ISOLDE. Following the outcome of our preparatory experiments, we have chosen $\mathrm{CNT}$ as target material. The ABRABLA code predicts the formation of ${ }^{8} \mathrm{~B}$ mainly by fragmentation of ${ }^{12} \mathrm{C}$ which is induced by impact of the $1.4 \mathrm{GeV}$ proton beam. A water-cooled transfer line ensured that only volatile compounds like $\mathrm{BF}_{3}$ could reach the VADIS ion source. A constant flow of $\mathrm{SF}_{6}$ was applied through a calibrated leak.

The first target unit tested in 2014 was equipped with a calibrated leak of $3.7 \times 10^{-5} \mathrm{mbar} \mathrm{L} / \mathrm{s}$ (air). Measurements started at a target temperature of $1350^{\circ} \mathrm{C}$ which was successively increased to a maximum of $2000^{\circ} \mathrm{C}$. The extracted radionuclides have been characterized with the ISOLDE tape station [35] located at the central beam line. The tape station allows measurements with a plastic scintillator in $4 \pi$ geometry and a high purity germanium gamma detector.

As shown in fig. $6,{ }^{8} \mathrm{~B}$ is known to undergo $\beta^{+} 2 \alpha$ decay, the isobaric ${ }^{8} \mathrm{Li}$, which is also produced in fragmentation reactions of ${ }^{12,13} \mathrm{C}$, decays by $\beta^{-} 2 \alpha$ [36]. Therefore, the annihilation radiation allows to distinguish between both isobars. In the investigated temperature range the scintillation counter indicated activity on mass 8 , reaching a maximum of $1 \times 10^{4} / \mu \mathrm{C}$ at $2000^{\circ} \mathrm{C}$. The half-life was determined to be $800 \pm 100 \mathrm{~ms}$. The annihilation peak in the gamma spectrum revealed, that $3 \%$ of the activity found by scintillation counts was originating from $\beta^{+}$emitters, leading to a ${ }^{8} \mathrm{~B}$ yield of $3 \times 10^{2} / \mu \mathrm{C}$. Potential isobaric contaminants on mass 8 are ${ }^{8} \mathrm{Li}$ and ${ }^{8} \mathrm{He}$. While lithium is expected to be retained in the water-cooled transfer line, the volatile helium nuclides are extracted as beam and reach the tape of the tape station. During tape transport from collection to measurement position $(900 \mathrm{~ms}){ }^{8} \mathrm{He}$ mostly decays to ${ }^{8} \mathrm{Li}$, the latter causing scintillator events while 


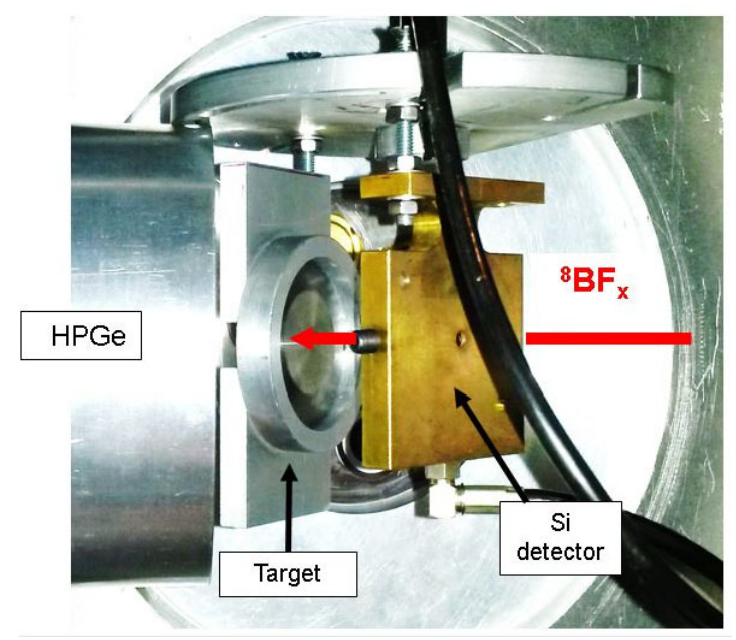

Fig. 7. In beam detection setup consisting of a silicon and high purity germanium detector.

the gamma radiation originating from the decay of ${ }^{8} \mathrm{He}$ is not seen. In contrast to offline studies, activity on the masses of ${ }^{8} \mathrm{BF},{ }^{8} \mathrm{BF}_{2},{ }^{8} \mathrm{BF}_{3}$ as well as ${ }^{8} \mathrm{BO},{ }^{8} \mathrm{BOC}$ and ${ }^{8} \mathrm{BOF}$ could not be found. The detection limit for ${ }^{8} \mathrm{~B}$ was calculated to be 75 ions per $\mu \mathrm{C}$. The concurrent absence of stable $\mathrm{SF}_{\mathrm{x}}$ and $\mathrm{TaF}_{\mathrm{x}}$ ions, along with the absence of $\mathrm{BF}_{\mathrm{x}}$ radioisotopes indicated that the formation of boron fluorides was limited by the availability of fluorine.

Therefore, we increased the size of the calibrated leak in the second unit to $1.8 \times 10^{-4} \mathrm{mbar} \mathrm{L} / \mathrm{s}$. It contained $14.96 \mathrm{~g}$ of carbon nanotubes pressed to pellets of $15 \mathrm{~mm}$ diameter, yielding an areal density of $8.4 \mathrm{~g} / \mathrm{cm}^{2}$. In order to strengthen the argument that the measured activity is caused by ${ }^{8} \mathrm{~B}$ we made use of an in-beam detection system (fig. 7) equipped with a silicon and a high purity germanium detector allowing the detection of alpha and gamma annihilation events in coincidence $(15 \mu \mathrm{s}$ window). In this experiment, the target was operated at $1500{ }^{\circ} \mathrm{C}$ as well as at $1750^{\circ} \mathrm{C}$. In contrast to the first measurement, beams of $\mathrm{BF}_{\mathrm{x}}(0 \leq \mathrm{x} \leq 3)$ radioisotopes could now be extracted and beams of stable sulfur fluorides were seen, indicating the availability of fluoride in the target. In agreement with the offline ionization tests, the yields were increasing in the series ${ }^{8} \mathrm{BF}_{3}<{ }^{8} \mathrm{BF}<{ }^{8} \mathrm{~B}<{ }^{8} \mathrm{BF}_{2}$. The yield found for ${ }^{8} \mathrm{BF}_{2}$ was calculated to be $6.4 \times 10^{4} / \mu \mathrm{C}$ at the target temperature of $1500{ }^{\circ} \mathrm{C}$. As expected, ${ }^{8} \mathrm{He}$ was only found on mass $8\left(4.5 \times 10^{3} / \mu \mathrm{C}\right)$ and nearly all activity registered by the scintillator on the ${ }^{8} \mathrm{BF}_{\mathrm{x}}$ masses could be assigned to a positron emitter.

Figure 8 shows a measured alpha spectrum in comparison with data obtained in a more sophisticated setup by Roger et al. [9] by implanting ${ }^{8} \mathrm{~B}$ at a rate of 7 ions/s in a finely segmented double sided silicon micro strip detector (DSSD). Roger et al. applied a correction for contributions of $\beta$-particles and $\alpha$-efficiency, which is not of importance for the unambiguous identification of ${ }^{8} \mathrm{~B}$, and therefore not considered in this work.

The high energy tails of both spectra are well in agreement. Minor deviations in the raising part of the spec-

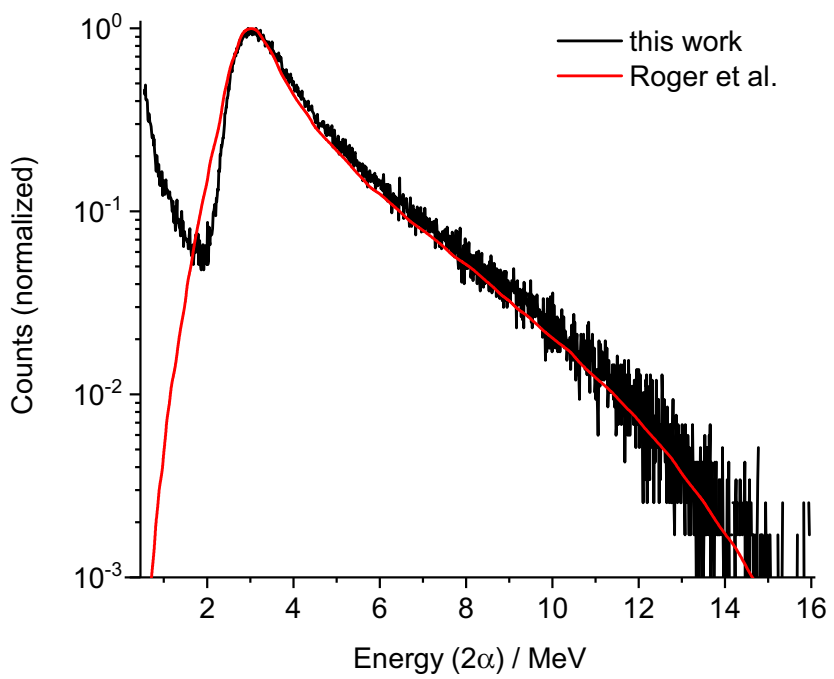

Fig. 8. Single alpha spectrum (black) recorded on the mass of ${ }^{8} \mathrm{BF}_{2}$ in comparison with two alpha spectrum taken by Roger et al. [9]. The respective energies of the single alpha spectrum have been multiplied by two to allow the comparison to the two alpha spectrum. The two alpha spectrum by Roger et al. is corrected for $\beta$ summing and $\alpha$ detection efficiency. These minor corrections are not necessary for the identification of ${ }^{8} \mathrm{~B}$ and therefore not included in this work.

tra can be explained by the low energy threshold for $\alpha$ particles in the setup, as well as electronic noise and contributions due to energetic $\beta$-particles. The consistence of both spectra along with registered annihilation radiation in coincidence with alpha events allows us to assign the activity to the decay of ${ }^{8} \mathrm{~B}$.

\subsection{Release properties}

The time-dependent release of ${ }^{8} \mathrm{BF}_{2}$ was studied with the scintillation counter of the ISOLDE tape station. In contrast to the offline mobility studies discussed in sect. 4 , it was crucial to also consider effusion effects due to the significantly shorter time scale. A release curve is obtained by collecting a small fraction of the released ions at a welldefined time $t$ after proton impact, determining the collected activity and repeating the procedure while varying $t$. Correcting this release curve by the decay losses during the extraction time $t$ yields a release curve for a stable isotope of the same species $p(t)$, since the diffusion and effusion properties only depend on the chemical species and not on the isotope.

The release curve for a stable isotope $p(t)$ is given by a folding integral of effusion contribution $p_{\nu} \propto e^{-t / t_{\text {eff }}}[4]$ and diffusion contribution $p_{\mu}=-\mathrm{d} f(t) / \mathrm{d} t$. For an agglomeration of fibers $p(t)$ is given by

$$
\begin{aligned}
p(t) & =\frac{1}{A_{K}} \int_{0}^{t} p_{\mu}\left(t^{\prime}\right) p_{\nu}\left(t-t^{\prime}\right) \mathrm{d} t^{\prime} \\
& =\frac{1}{A_{K}} \sum_{m=1}^{\infty} \frac{\mathrm{e}^{-c_{m} t / \tau_{D}}-\mathrm{e}^{-t / t_{\mathrm{eff}}}}{\tau_{D}-c_{m} t_{\mathrm{eff}}}
\end{aligned}
$$




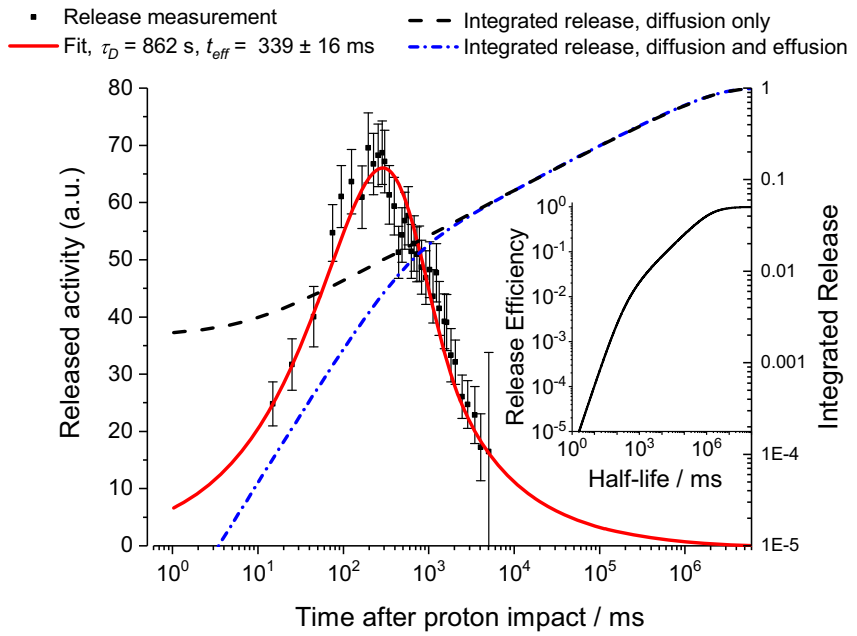

Fig. 9. Released activity versus time $t$ after proton impact, measured with the scintillation detector of the ISOLDE tape station on the mass of ${ }^{8} \mathrm{BF}_{2}$ and corrected for the decay during release. Inset: release efficiency $y\left(T_{1 / 2}\right)$, derived from the measured release curve and offline mobility studies (see text).

where the normalization parameter $A_{K}$ is chosen such that $p(t)$ integrates to unity and computes to $A_{K}=\sum_{m=1}^{\infty} c_{m}^{-1}$ Folding the release function of the stable isotope $p(t)$ with the decay losses of a radioactive isotope $\left(T_{1 / 2}\right)$ gives the fraction of released isotopes, the so called release efficiency $y$, which computes to

$$
\begin{aligned}
& y\left(T_{1 / 2}\right)=\int_{0}^{\infty} p(t) \mathrm{e}^{-\ln (2) t / T_{1 / 2}} \mathrm{~d} t= \\
& \frac{1}{A_{K}} \sum_{m=1}^{\infty} \frac{T_{1 / 2}^{2}}{\left(c_{m} T_{1 / 2}+\tau_{D} \ln (2)\right)\left(T_{1 / 2}+t_{\text {eff }} \ln (2)\right)} .
\end{aligned}
$$

The experimentally obtained release curve $p(t)$ is shown in fig. 9. The function following eq. (5) does not exhibit a strong constraint in the parameter $\tau_{D}$. Therefore, the result obtained in the offline mobility studies was used, which is in agreement with the experimental data and allows us to determine the effusion time constant to be $t_{\text {eff }}=339 \pm 16 \mathrm{~ms}$. The expression used for the effusion contribution to the release function $p_{\nu}(t)$ holds for the effusion of uniformly distributed particles in a certain volume through a small orifice. However, the ISOLDE target container is connected via a transfer line to the ion source, which causes a delayed release. To account for the slower release, a second effusion time parameter leading to $p_{\nu}(t) \propto\left(1-\mathrm{e}^{-t / t_{\mathrm{eff} 1}}\right) \mathrm{e}^{-t / t_{\mathrm{eff} 2}}$ was introduced in [37] based on the result of Monte Carlo simulations and was applied in [38]. In the case analyzed here, the second parameter did not improve the fit significantly and was therefore discarded.

To justify that effusion processes were neglected at high temperatures within the deduction of $\tau_{D}$ from offline mobility experiments, fig. 9 shows the integrated release according to eq. (4), where effusion is not considered, as well as the integration of the experimental release curve fitted by eq. (5) and therefore taking into account effu- sion. It can be seen from fig. 9 that at the heat treatment time of 30 minutes $\left(1.8 \times 10^{6} \mathrm{~ms}\right)$ effusion contributions are negligible. Also shown as insert in fig. 9 is a plot of the release efficiency (eq. (6)). The release efficiency of the investigated isotope ${ }^{8} \mathrm{~B}$ computes to $1.7 \%$ $\left(T_{1 / 2}=770 \mathrm{~ms}\right)$, the release efficiencies of the isotopes ${ }^{12} \mathrm{~B}\left(T_{1 / 2}=20.2 \mathrm{~ms}\right)$ and ${ }^{13} \mathrm{~B}\left(T_{1 / 2}=17.3 \mathrm{~ms}\right)$ are expected to be $0.029 \%$ and $0.023 \%$, respectively. Comparing the measured yield weighted by the release fraction with the in-target production predicted by ABRABLA, indicates an overall efficiency for $\mathrm{BF}_{2}$ molecular formation, ionization and transport of $\epsilon_{\text {irrev }} \epsilon_{\text {form }} \epsilon_{\text {ion }}=1.1 \%$, which is close to the efficiency value of $1.5 \%$ obtained in offline studies. Assuming in-target production yields predicted by FLUKA [39], the same efficiency product computes to $0.15 \%$. The availability of this information allows to predict yields of the more exotic boron isotopes ${ }^{12,13} \mathrm{~B}$, which are given in table 3 .

For practical purposes, ISOLDE release curves are typically fitted with the three exponential function given in eq. (7) and discussed in ref. [40].

$$
\begin{aligned}
p(t)= & \frac{1}{A_{L}}\left(1-\mathrm{e}^{-t / t_{\text {rise }}}\right) \\
& \cdot\left(\alpha \mathrm{e}^{-t / t_{\text {fall1 }}}+(1-\alpha) \mathrm{e}^{-t / t_{\text {fall } 2}}\right) .
\end{aligned}
$$

The release properties obtained from the curve fit have been evaluated to be as follows for the release of a stable isotope.

\begin{tabular}{lcccc} 
Ion & $t_{\text {rise }}$ & $\alpha$ & $t_{\text {fall1 }}$ & $t_{\text {fall } 2}$ \\
\hline${ }^{8} \mathrm{BF}_{2}+$ & $52 \mathrm{~ms}$ & 0.20 & $253 \mathrm{~ms}$ & $3015 \mathrm{~ms}$
\end{tabular}

\subsection{Further developments and outlook}

Another test of the target unit took place in 2016 aiming at post-acceleration of the low energy ${ }^{8} \mathrm{~B}$ beam at HIEISOLDE [41]. In 2017, the first ${ }^{8} \mathrm{~B}$ ISOL beams were delivered to a physics experiment (IS633) [42], investigating the electron capture of ${ }^{8} \mathrm{~B}$ into highly excited states in ${ }^{8} \mathrm{Be}$ using the ISOLDE decay station [43]. In 2018, the first accelerated ${ }^{8} \mathrm{~B}$ beams $(4.9 \mathrm{MeV} / \mathrm{u})$ were delivered via HIE-ISOLDE to study reaction dynamics of proton-halo induced collisions [44]. Instead of sulfur hexafluoride, the fluorinating agent tetrafluoromethane $\left(\mathrm{CF}_{4}\right)$ was used to avoid a possible sulfur contamination of the target station. After injection of $\mathrm{CF}_{4}$, a stable isobaric contamination on the mass of ${ }^{8} \mathrm{BF}_{2}$ of ca. $2 \mathrm{nA}$ was found in the low energy beam, which exceeds the typical acceptance limit of the high energy setup for efficient operation. The recorded mass spectra show molybdenum fluoride beams, and indicate that the contamination is arising from doubly charged ${ }^{92}$ Mo. Parts of the VADIS ion sources are build of molybdenum, thus serving as molybdenum source. The development of a molybdenum-free ion source could increase the efficiency and beam yields for HIE-ISOLDE. 
Table 3. Measured $\left({ }^{8} \mathrm{BF}_{2}\right)$ and predicted $\left({ }^{12,13} \mathrm{BF}_{2}\right)$ yields of radioactive boron beams based on the release efficiency function $y\left(T_{1 / 2}\right)$ deduced from the measured release curve of ${ }^{8} \mathrm{BF}_{2}$, the ionization and extraction efficiency as well as in-target production cross sections computed by Monte Carlo codes.

\begin{tabular}{|c|c|c|c|c|c|}
\hline Boron isotope & \multicolumn{2}{|c|}{8} & \multicolumn{2}{|c|}{12} & 13 \\
\hline Half-Life & \multicolumn{2}{|c|}{$770 \mathrm{~ms}$} & \multicolumn{2}{|c|}{$20.20 \mathrm{~ms}$} & $17.33 \mathrm{~ms}$ \\
\hline Release Efficiency & \multicolumn{2}{|c|}{$1.7 \%$} & \multicolumn{2}{|c|}{$0.029 \%$} & $0.023 \%$ \\
\hline Code & ABRABLA & FLUKA & ABRABLA & FLUKA & FLUKA \\
\hline In-target production $/ \mu \mathrm{C}$ & $3.3 \times 10^{8}$ & $2.4 \times 10^{9}$ & $2.2 \times 10^{8}$ & $7.3 \times 10^{8}$ & $5.2 \times 10^{6}$ \\
\hline Beam yield $/ \mu \mathrm{C}$ & \multicolumn{2}{|c|}{$6.4 \times 10^{4}$} & $7.1 \times 10^{2 \mathrm{a}}$ & $3.2 \times 10^{2 \mathrm{a}}$ & $1.9 \times 10^{0 \mathrm{a}}$ \\
\hline
\end{tabular}

\section{Conclusions}

Following the experimental and calculation strategy shown here, we successfully developed and tested a target unit for the production of exotic boron ISOL beams. By using multiwalled carbon nanotubes we could achieve high mobility of boron inside the grains of the target material, which leads to a fast extraction of boron and therefore relatively small losses due to decay during the diffusion process. The low volatility and high reactivity of boron, which hampers the release in its atomic form could be overcome by the extraction as volatile molecule. We have chosen fluorides as volatile carriers for boron, which are produced in-situ by injection of sulfur hexafluoride into the target container. The compound was ionized in an arc discharge electron impact VADIS ion source which was equipped with a water cooled copper transfer line to retain condensible elements and compounds. In online experiments yields of $6.4 \times 10^{4}{ }^{8} \mathrm{BF}_{2}$ ions per $\mu \mathrm{C}$ of protons could be determined while an average proton intensity of up to $2 \mu \mathrm{A}$ is available at ISOLDE [1]. The attainable yields drastically exceed yields reported to date from thin-target facilities and therefore pave the way for new physics experiments.

We would like to thank the radio protection staff of CERN DGS/RP-DC, P. Carbonez, A. Chantelauze and G. Lagneau, for hosting the setup used for offline mobility studies and the possibility to use the radioactive sources. We also acknowledge support from the ISOLDE operating staff for separator setup and support during the online experiment, the ISOLTRAP team for their effort to investigate the beam composition, as well as R. Dos Santos Augusto for support regarding the FLUKA code, and A. Kelic-Heil for ABRABLA support. This work has been partly supported by the Spanish CICYT through projects, FPA2015-64969-P, and by the European Union by means of the European Commission within its Seventh Framework Program (FP7) via ENSAR (Contract No. 262010). Further, this project has received funding from the European Union Horizon 2020 research and innovation program under grant agreement No. 654002. One of the authors, CS, acknowledges support by the German BMBF via the Gentner program and his local project 05P12RDCIA.

\section{Author Contribution Statement}

All the authors were involved in the preparation of the manuscript. All authors have read and approved the final manuscript.

Data Availability Statement This manuscript has no associated data or the data will not be deposited. [Authors' comment: All data generated during this study are contained in this published article.]

Publisher's Note The EPJ Publishers remain neutral with regard to jurisdictional claims in published maps and institutional affiliations.

Open Access This is an open access article distributed under the terms of the Creative Commons Attribution License (http://creativecommons.org/licenses/by/4.0), which permits unrestricted use, distribution, and reproduction in any medium, provided the original work is properly cited.

\section{References}

1. R. Catherall, W. Andreazza, M. Breitenfeldt, A. Dorsival, G.J. Focker, T.P. Gharsa, T. Giles, J.L. Grenard, F. Locci, P. Martins et al., J. Phys. G: Nucl. Part. Phys. 44, 094002 (2017).

2. U. Köster, P. Carbonez, A. Dorsival, J. Dvorak, R. Eichler, S. Fernandes, H. Frånberg, J. Neuhausen, Z. Novackova, R. Wilfinger et al., Eur. Phys. J. ST 150, 285 (2007).

3. C. Seiffert, Production of radioactive molecular beams for CERN-ISOLDE, Doctoral Thesis, Technische Universität Darmstadt, Germany (2014) http://cds.cern.ch/ record/2064456.

4. R. Kirchner, Nucl. Instrum. Methods Phys. Res. Sect. B 70, 186 (1992).

5. J. Ramos, A. Gottberg, T. Mendonça, C. Seiffert, A. Senos, H. Fynbo, O. Tengblad, J. Briz, M. Lund, G. Koldste et al., Nucl. Instrum. Methods Phys. Res. Sect. B 320, 83 (2014). 
6. A. DiPietro, P. Figuera, A. Bonaccorso, M. Fisichella, J. Gomez-Camacho, M. Lattuada, M. Milin, A. Musumarra, M. Pellegriti, D. Santonocito et al., Tech. Rep. CERNINTC-2010-063. INTC-I-126, CERN, Geneva (2010) https://cds. cern.ch/record/1298251.

7. M. Pellegriti, P. Descouvemont, A. DiPietro, P. Figuera, M. Fisichella, M. Lattuada, M. Milin, A. Musumarra, V. Scuderi, D. Torresi et al., Tech. Rep. CERN-INTC2010-069. INTC-I-127, CERN, Geneva (2010) https:// cds.cern.ch/record/1298603.

8. Sacavm Lisbon Leuven Göttingen Porto Durban CERN Collaboration, EC-SLI Collaboration (U. Wahl, L. Amorim, J.P. Araujo, K. Bharuth-Ram, J.G. Correia, M.R. da Silva, S. Decoster, H. Hofsäss, M. Nagl, L. Pereira et al.), Tech. Rep. CERN-INTC-2010-077. INTC-I-130, CERN, Geneva (2010) https://cds.cern.ch/record/ 1298732 .

9. T. Roger, J. Büscher, B. Bastin, O.S. Kirsebom, R. Raabe, M. Alcorta, J. Äystö, M.J.G. Borge, M. CarmonaGallardo, T.E. Cocolios et al., Phys. Rev. Lett. 108, 162502 (2012).

10. O.S. Kirsebom, S. Hyldegaard, M. Alcorta, M.J.G. Borge, J. Büscher, T. Eronen, S. Fox, B.R. Fulton, H.O.U. Fynbo, H. Hultgren et al., Phys. Rev. C 83, 065802 (2011).

11. J.R. Rumble (Editor), CRC Handbook of Chemistry and Physics: A Ready-Reference Book of Chemical and Physical Data, 99th edn. (Taylor \& Francis, 2018).

12. A. Kelic, M.V. Ricciardi, K.H. Schmidt, ABLA07 - towards a complete description of the decay channels of a nuclear system from spontaneous fission to multifragmentation, in Proceedings of the Joint ICTP-IAEA Advanced Workshop on Model Codes for Spallation Reactions, Trieste, Italy, February 4-8, 2008 (IAEA, 2008) arXiv:0906.4193.

13. T. Bjørnstad, E. Hagebø, P. Hoff, O. Jonsson, E. Kugler, H. Ravn, S. Sundell, B. Vosicki, Nucl. Instrum. Methods Phys. Res. Sect. B 26, 174 (1987).

14. W. Callister, Materials Science and Engineering: An Introduction (John Wiley \& Sons, New York, 2007).

15. M. Fujioka, Y. Arai, Nucl. Instrum. Methods Phys. Res. Sect. B 186, 409 (1981).

16. H. Fechtig, S. Kalbitzer, The Diffusion of Argon in Potassium-Bearing Solids (Springer Berlin Heidelberg, Berlin, Heidelberg, 1966) pp. 68-107.

17. D. Fink, L. Wang, Radiat. Effects Defects Solids 114, 343 (1990).

18. D. Brown, M. Chadwick, R. Capote, A. Kahler, A. Trkov, M. Herman, A. Sonzogni, Y. Danon, A. Carlson, M. Dunn et al., Nucl. Data Sheets 148, 1 (2018).

19. A. Göpfert, F.J. Hambsch, H. Bax, Nucl. Instrum. Methods Phys. Res. Sect. A 441, 438 (2000).

20. L.W. Weston, J.H. Todd, Nucl. Sci. Eng. 109, 113 (1991).

21. J. Vacik, V. Hnatowicz, J. Cervena, S. Posta, U. Köster, G. Pasold, AIP Conf. Proc. 1099, 836 (2009).

22. J. Ramos, A. Senos, T. Stora, C. Fernandes, P. Bowen, J. Eur. Ceramic Soc. 37, 3899 (2017).

23. G. Heller, Gmelin Handbook of Inorganic and Organometallic Chemistry - B Boron Compounds, 4th Supplement, Boron and Oxygen (Springer Berlin Heidelberg, 1991) https://doi.org/10.1007/978-3-662-06150-3.
24. G. Henning, J. Chem. Phys. 42, 1167 (1965).

25. Y.V. Novak, T.V. Pyrkova, A.F. Kuteinikov, Khimiya Tverdogo Topliva 16, 127 (1982).

26. J.F. Ziegler, M. Ziegler, J. Biersack, Nucl. Instrum. Methods Phys. Res. Sect. B 268, 1818 (2010).

27. J.F. Ziegler, SRIM The Stopping Range of Ions in Matter, http://www.srim.org (2018) (accessed 24 August 2018).

28. J.P. Ramos, Thick solid targets for the production and online release of radioisotopes: the importance of the material characteristics - A Review, in preparation.

29. A.I. Skoulidas, D.M. Ackerman, J.K. Johnson, D.S. Sholl, Phys. Rev. Lett. 89, 185901 (2002).

30. A. Noy, J. Phys. Chem. C 117, 7656 (2013).

31. G.A. Perkova, A.V. Demin, E.F. Chalykh, Y.M. Kachanov, Khimiya Tverdogo Topliva 5, 163 (1976).

32. A. Roine, HSC Chemistry 7.1, http://www.hscchemistry.net (2010) (accessed 19 July 2015).

33. L. Penescu, R. Catherall, J. Lettry, T. Stora, Rev. Sci. Instrum. 81, 02 A906 (2010).

34. Nucleonica GmbH, Nucleonica Nuclear Science Portal, www. nucleonica.com (2017) version 3.0.65.

35. M. Turrión, M. Eller, R. Catherall, L. Fraile, U. HermanIzycka, U. Köster, J. Lettry, K. Riisager, T. Stora, Nucl. Instrum. Methods Phys. Res. Sect. B 266, 4674 (2008).

36. W.T. Winter, S.J. Freedman, K.E. Rehm, J.P. Schiffer, Phys. Rev. C 73, 025503 (2006).

37. E. Bouquerel, Atomic beam merging and suppression of alkali contaminants in multi body high power targets: Design and test of target and ion source prototypes at isolde, Doctoral Thesis (2009).

38. T. Stora, E. Noah, R. Hodak, T.Y. Hirsh, M. Hass, V. Kumar, K. Singh, S. Vaintraub, P. Delahaye, H. FranbergDelahaye et al., EPL 98, 32001 (2012).

39. A. Ferrari, P.R. Sala, A. Fass, J. Ranft, FLUKA: A multiparticle transport code (program version 2005), CERN Yellow Reports: Monographs (CERN, Geneva, 2005) http:// cds. cern. ch/record/898301.

40. J. Lettry, R. Catherall, P. Drumm, P. Van Duppen, A. Evensen, G. Focker, A. Jokinen, O. Jonsson, E. Kugler, H. Ravn et al., Nucl. Instrum. Methods Phys. Res. Sect. B 126, 130 (1997).

41. Y. Kadi, Y. Blumenfeld, W.V. Delsolaro, M.A. Fraser, M. Huyse, A.P. Koufidou, J.A. Rodriguez, F. Wenander, J. Phys. G: Nucl. Part. Phys. 44, 084003 (2017).

42. M. Borge, J. Cederkall, P. Diaz Fernandez, L. Fraile, H. Fynbo, A. Heinz, J. Jensen, H. Johansson, B. Jonson, O. Kirsebom et al., Tech. Rep. CERN-INTC-2016-052. INTCP-482, CERN, Geneva (2016) http://cds.cern.ch/ record/2222324.

43. ISOLDE-IDS Setup, http://isolde-ids.web.cern.ch/ isolde-ids/\#setup (2018) (accessed 31 July 2018).

44. A. Di Pietro, P. Figuera, J. Ballof, A. Bonaccorso, J. Cederkall, T. Davinson, J. Fernandez Garcia, M. Fisichella, M. Garcia Borge, J. Gomez Camacho et al., Tech. Rep. CERN-INTC-2016-018. INTC-P-463, CERN, Geneva (2016) https://cds.cern.ch/record/2120157. 